\title{
Uso de substâncias psicoativas em Serviço de Urgência em Psiquiatria da Infância e Adolescência de Belo Horizonte
}

\author{
Psychoactive substance use at an Emergency Department in Childhood and \\ Adolescence Psychiatry service in Belo Horizonte
}

\author{
Amanda Carvalho Mitre Chaves ${ }^{1}$, Ana Clara Brant Moreira Ferreira ${ }^{1}$, Ana Maria Soares \\ Lacerda $^{1}$, Marina Sad Navarro ${ }^{1}$, Gabriela Peixoto Campos ${ }^{1}$, Julia Passini Vaz-Tostes ${ }^{1}$, Ana \\ Luisa Souto Gandra ${ }^{1}$, Ana Maria Fidelis Silva Campos ${ }^{1}$, Luciana Rodrigues da Cunha ${ }^{2}$, \\ Ana Carolina Lobato Machado ${ }^{2}$
}

Chaves ACM, Ferreira ACBM, Lacerda MAS, Navarro MS, Campos GP, Tostes JPV, Gandra ALS, Campos AMFS, Cunha LR, Machado MCL. Uso de substâncias psicoativas em Serviço de Urgência em Psiquiatria da Infância e Adolescência de Belo Horizonte / Psychoactive substance use at an Emergency Department in Childhood and Adolescence Psychiatry Service in Belo Horizonte. Rev Med (São Paulo). 2020 jul.-ago.;99(4):342-9.

RESUMO: Introdução: O uso de álcool, tabaco e drogas ilícitas por crianças e adolescentes é um problema de saúde pública que assume importância cada vez maior em todo o mundo. O aumento da prevalência do uso dessas substâncias foi acompanhado por maiores níveis de ansiedade, sintomas depressivos e outros transtornos psiquiátricos, além de aumento nas taxas de violência e mortalidade. Objetivo: Analisar a prevalência de atendimentos por uso de drogas em um Serviço de Urgência em Psiquiatria da Infância e Adolescência de Belo Horizonte, bem como determinar o perfil sociodemográfico desses pacientes. Metodologia: Trata-se de estudo transversal realizado por meio de revisão de prontuários de pacientes que procuraram um serviço de urgência em saúde mental infantil durante o período de um ano. A análise comparativa das variáveis categóricas foi feita pelo teste qui-quadrado de Pearson ou exato de Fisher. Foi utilizado o teste t de Student de amostras independentes para a variável contínua. Resultados: Foram coletados dados de 2255 atendimentos. A prevalência do uso de substâncias psicoativas encontrada foi de $17,7 \%$ da amostra. A droga mais utilizada foi a maconha (14,1\%), seguida por cocaína (7\%), álcool $(5,7 \%)$, tabaco $(4,2 \%)$, solventes e inalantes $(3,6 \%)$ e crack $(1,7 \%)$. Foi observado maior consumo em homens, faixa etária 15-17 anos e etnia parda. Conclusão: Foi observada significativa prevalência no uso de drogas entre crianças e adolescentes. É possível presumir que a real prevalência seja maior, uma vez que se acredita que a porcentagem real de jovens que utilizam essas substâncias é maior que a parcela que chega ao serviço de urgência. Os resultados encontrados demonstram uma necessidade de reforçar estratégias de intervenções preventivas para essa população.

Descritores: Adolescente; Criança; Saúde mental; Transtornos relacionados ao uso de substâncias.
ABSTRACT: Introduction: The use of alcohol, tobacco and illicit drugs by children and teenagers is a growing public health problem worldwide. The increased prevalence of substance use was associated with higher rates of anxiety, depressive symptoms and other psychiatric disorders, as well as a rise in mortality and violence rates. Objective: To analyze the prevalence of drug-related care at a Childhood and Adolescent Psychiatric Emergency Department in Belo Horizonte and to determine the sociodemographic profile of these patients. Methods: This is a cross-sectional study conducted through the review of medical records of patients who sought an emergency service of child mental health during the period of one year. A comparative analysis of strategic variables was performed using Pearson's square test of Fisher's exact test. Student's T Test was used for continuous variable. Results: data from 2255 medical records were assessed. A prevalence of psychoactive substance use of $17.7 \%$ of the appointments was found. The most used drug was marijuana $(14.1 \%)$, followed by cocaine $(7 \%)$, alcohol $(5.7 \%)$, tobacco $(4.2 \%)$, solvents and inhalants $(3.6 \%)$ and crack $(1.7 \%)$. Higher consumption was observed in men, age 15-17 years and brown ethnicity. Conclusions: A significant prevalence of drug use was observed among child and adolescents. It is possible to assume that the real prevalence is even higher, since it is believed that the real percentage of young people who use these substances is greater than the portion that arrives at emergency service. The results of this study demonstrate a necessity of reinforcing preventive intervention strategies for this population.

Keywords: Adolescent; Child; Mental health; Substance-related disorders.

1. Acadêmicas do curso de medicina da Faculdade Ciências Médicas de Minas Gerais, Belo Horizonte, MG. ORCID: Chaves ACM - https://orcid. org/0000-0001-5137-2080; Ferreira ACBM - https://orcid.org/0000-0002-3581-2506; Gandra ALS - https://orcid.org/0000-0001-6962-8494; Campos AMF - https://orcid.org/0000-0002-0494-9913; Lacerda AMS - https://orcid.org/0000-0001-8093-0600; Vaz-Tostes JP - https://orcid.org/0000-00025122-4114; Campos GP - https://orcid.org/0000-0002-6584-0401; Navarro MS - https://orcid.org/0000-0002-1016-9518. E-mail: amandamitrechaves@, gmail.com; anacbrant11@gmail.com; analuisagandra@hotmail.com; anamariafidelissilvacampos@gmail.com; anamarialacerda14@gmail.com; jupvtostes0812@gmail.com; gabrielapeixoto8@hotmail.com; marina_sadn@hotmail.com.

2. Centro Psíquico da Adolescência e Infância, Belo Horizonte, MG. ORC̄ID: Cunha LR - https://orcid.org/0000-0001-7699-8717; Machado MCL https://orcid.org/0000-0001-5006-6129. E-mail: lobatocarol@yahoo.com.br; 1rcunha@gmail.com.

Endereço para correspondência: Amanda Carvalho Mitre Chaves. Avenida Carandaí, № 78. Bairro: Funcionários, Belo Horizonte, MG. CEP: 30130060.

E-mail: amandamitrechaves@gmail.com. 


\section{INTRODUÇÃO}

$\mathrm{O}$ uso de substâncias psicoativas (SPAS) é difundido e endêmico entre adolescentes ${ }^{1}$. Nos Estados Unidos, quase metade dos adolescentes já experimentou uma droga ilícita e mais de $80 \%$ já utilizou álcool ${ }^{1}$. Muitos sofrem consequências negativas sociais, na saúde física e emocional, como sentimentos de tristeza, solidão, insônia e ideação suicida ${ }^{1,2}$.

Estudos brasileiros revelam que a prevalência de crianças e adolescentes que já consumiram álcool é de aproximadamente $60 \%$, e que $25,5 \%$ já utilizaram outras drogas diferentes de álcool e tabaco ${ }^{2,3}$. São sugeridos alguns fatores associados, como o uso de substâncias por familiares e amigos, pobreza, violência comunitária e/ou escolar, uso de drogas e/ou álcool pelos pais, exposição a drogas e/ou álcool intrauterinos, conflito conjugal, disfunção familiar e/ou ambiente familiar perturbado, maus-tratos, abuso infantil, e alguns fatores individuais como irritabilidade, dificuldades motoras, de linguagem e cognitivas e comportamento agressivo precoce ${ }^{1,2}$.

Como consequência, há relatos de altas taxas de transtornos mentais concomitantes ao uso (61 a 88\%) entre adolescentes de 13 a 18 anos, como transtorno de conduta, transtorno de déficit de atenção e hiperatividade (TDAH), transtorno depressivo maior, transtorno bipolar, transtorno de estresse pós-traumático, transtornos de ansiedade e esquizofrenia ${ }^{1}$. McCardle $\mathrm{e}^{4}$ corrobora, de certa forma, essa estatística quando relata que o aumento da prevalência do uso de drogas ocorreu em paralelo com o aumento das taxas de ansiedade e sintomas depressivos e de mortes relacionadas ao uso indevido de substâncias. Ademais, conclui que os transtornos por uso de substâncias são potencialmente tratáveis e devem ser gerenciados como doenças crônicas recidivantes de origem complexa ${ }^{4}$.

Além disso, o uso de drogas está relacionado ao aumento dos níveis de violência urbana, como homicídios, agressões e acidentes de trânsito, e também no ambiente familiar, caracterizando a violência doméstica ${ }^{5}$. Estudo realizado na cidade de Belo Horizonte revelou que, de 2000 a 2009, cerca de $30 \%$ dos óbitos por homicídio na população residente em Belo Horizonte apresentaram álcool, maconha, cocaína ou crack no exame post mortem ${ }^{6}$. Assim, o prejuízo provocado pelo uso de substâncias não envolve somente o indivíduo, mas também traz consequências danosas em outros ambientes frequentados como trabalho, escola e meio social no geral.

Dessa forma, destaca-se a importância de se conhecer o perfil da população sob risco visando traçar estratégias de prevenção e intervenção precoces e buscando minimizar riscos relacionados à saúde do usuário e de todos os envolvidos, bem como questões socioeconômicas consequentes.

\section{MATERIAIS E MÉTODOS}

\section{Delineamento}

Trata-se de um estudo descritivo transversal, realizado por meio de dados de prontuários de pacientes atendidos na urgência de um Serviço de Saúde Mental da Infância e da Adolescência de Belo Horizonte. É um braço do estudo intitulado "Perfil de pacientes atendidos em um serviço de urgência em psiquiatria da infância e adolescência da cidade de Belo Horizonte", com foco nos atendimentos relacionados ao uso de álcool e outras drogas.

\section{Amostra}

Foram avaliados todos os atendimentos na urgência desse serviço no período compreendido entre 01 de junho de 2017 a 31 de maio de 2018 em busca de dados de atendimentos relacionados a uso de drogas, ou seja, em que o uso da substância tenha sido referido, pelo paciente ou responsável, como um dos motivos de busca de atendimento na urgência. Dessa forma, não foi utilizado cálculo amostral, sendo a amostra final composta pelos 2255 atendimentos ocorridos no período. A coleta de dados seguiu o roteiro do projeto original e, identificados os atendimentos de interesse para o estudo atual, foi feita a análise do perfil. Foram excluídos da pesquisa os prontuários que apresentavam dados ilegíveis.

\section{Instrumentos}

Para guiar a coleta de dados dos prontuários, foi utilizado um roteiro, elaborado pelos autores, para coleta de informações a respeito de dados sociodemográficos, história prévia ou atual de outro transtorno psiquiátrico, além de história do uso de substâncias. Todos os dados foram obtidos por meio de avaliação dos prontuários registrados por profissionais da área da saúde mental infanto-juvenil. O sexo e a idade foram coletados da ficha cadastral no prontuário que é feita com base nos documentos do paciente. Somente os diagnósticos psiquiátricos realizados pelos próprios especialistas do centro em questão foram incluídos na análise.

\section{Procedimentos}

A coleta de dados foi iniciada após aprovação pelo Comitê de Ética em Pesquisa. Os princípios de ética foram respeitados e estão de acordo com a Resolução 466/12 do Conselho Nacional de Saúde. O projeto foi aprovado dia 01 de junho de 2018, sendo o número do parecer 2.687.203 e CAAE 83419717.0.0000.5134.

O estudo foi conduzido no Centro Psíquico da Infância e Adolescência (CEPAI), instituição que presta atendimento multiprofissional em saúde mental da infância e adolescência, nos níveis secundário e terciário, para a população municipal e estadual. Assim, há atendimentos nas modalidades de ambulatório, hospital dia, internação e atendimento de urgência 24 horas. A porta de entrada 
dos pacientes no serviço é pelo acolhimento e/ou pelo atendimento psiquiátrico de urgência. A partir daí, é definida a modalidade de atendimento dos usuários no serviço e, após estabilização do quadro clínico, a equipe inicia o processo de transferência de cuidados para o território conforme preconizado no SUS.

Para a análise de dados, foi considerado como uso de substâncias psicoativas (SPA) a declaração de uso registrada no prontuário como uma das queixas relacionadas ao motivo da busca pelo atendimento. Para a definição de drogas e substâncias psicoativas, foi utilizada como referência a orientação da $\mathrm{OMS}^{7}$ : droga é qualquer substância que altera os processamentos bioquímicos ou fisiológicos dos tecidos ou organismos. No entanto, o termo é comumente usado para se referir às drogas psicoativas, ou seja, aquelas que interferem no psiquismo. As drogas lícitas são substâncias que podem causar dependência, mas são comercializadas legalmente (podendo haver restrição de uso). Já as drogas ilícitas não podem ser produzidas, fabricadas, adquiridas, comercializadas, fornecidas ou armazenadas por lei e, no Brasil, estão numeradas na Lista de Substâncias Sujeitas a Controle Especial da ANVISA ${ }^{8}$. As substâncias consideradas na avaliação foram álcool, maconha, cocaína, tabaco, crack, solventes e inalantes. Demais drogas ilícitas foram incluídas na categoria "outras SPA".

A análise de prontuários foi feita manualmente por meio da avaliação de arquivos físicos e online na plataforma da instituição e os dados foram tabulados para a análise de dados.

\section{Análise estatística}

A análise de caracterização dos dados foi feita com base nas frequências absolutas e porcentagens para as variáveis categóricas. Para as variáveis contínuas, foi utilizado o cálculo de média e desvio-padrão. A análise comparativa das variáveis categóricas foi feita pelo teste qui-quadrado de Pearson ou exato de Fisher, quando apropriado. Para as variáveis contínuas, foi utilizado o teste $t$ de Student de amostras independentes. A análise estatística foi feita no Statistical Package for Social Sciences [SPSS (IBM, Estados Unidos)] versão 20.0. O nível de significância considerado para todas as análises foi de $\mathrm{p}<0,05]$.

\section{RESULTADOS}

Foi obtida uma amostra composta por 2255 atendimentos, sendo $62,7 \%$ do sexo masculino e $37,3 \%$ do sexo feminino, média de idade $13.0 \pm 3.8$. Foi relatado uso de alguma substância psicoativa (SPA) em 400 atendimentos $(17,7 \%)$. A droga mais relatada foi a maconha $(14,1 \%)$, seguida por cocaína $(7 \%)$, álcool $(5,7 \%)$, tabaco $(4,2 \%)$, solventes e inalantes $(3,6 \%)$ e crack $(1,7 \%)$ (Tabela 1).
Tabela 1 - Prevalência de uso de SPA nos jovens atendidos no serviço de urgência

\begin{tabular}{ll}
\hline Uso de SPA & N (\%) \\
\hline Uso de Maconha & $318(14.1)$ \\
Sim & $1937(85.9)$ \\
Não & \\
Uso de Solventes e Inalantes & $81(3.6)$ \\
Sim & $2174(96.4)$ \\
Não & \\
Uso de Crack & $39(1.7)$ \\
Sim & $2216(98.3)$ \\
Não & \\
Uso de Cocaína & $157(7.0)$ \\
Sim & $2098(93.0)$ \\
Não & \\
Uso de Álcool & $129(5.7)$ \\
Sim & $2126(94.3)$ \\
Não & \\
Uso de Tabaco & $95(4.2)$ \\
Sim & $2160(95.8)$ \\
Não & \\
Uso de outros SPA & $30(1.3)$ \\
Sim & $2225(98.7)$ \\
Não &
\end{tabular}

O uso foi predominante no sexo masculino $(70,2 \%)$ (Figura 1). Entre todos os meninos avaliados, $20 \%$ possuíam relato de consumo de SPA, enquanto entre as meninas a proporção foi de 14\%. A média de idade dos indivíduos que usam SPA é $15.5 \pm 1.7$ e a faixa etária de maior consumo é $15-17$ anos (75,5\%), seguida por idade igual ou menor a 14 anos $(23,8 \%)$ (Tabela 2). Dentre todos os atendimentos, os pacientes usuários de SPA correspondem a 29,2\% dos indivíduos na faixa etária de 15-17 anos, em contraste com $7,8 \%$ dos pacientes com 14 anos ou menos $(p<0,001)$.

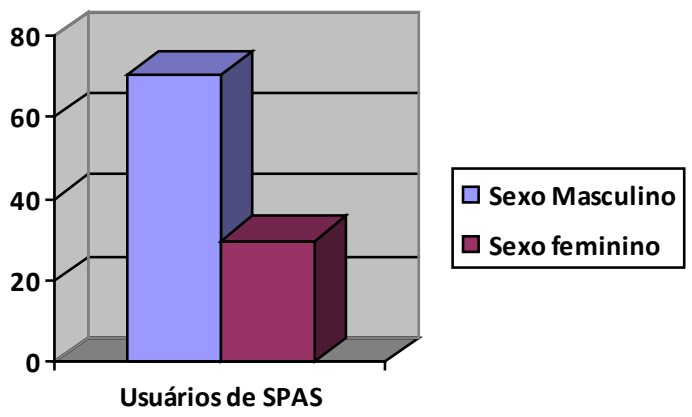

Figura 1 - Distribuição dos usuários de SPA de acordo com o sexo 
Tabela 2 - Frequência de uso de SPA por idade

\begin{tabular}{ll}
\hline \multicolumn{1}{l}{ Idade codificada } & \\
\hline$\leq 14$ anos & $95(23.8)$ \\
$15-17$ anos & $302(75.5)$ \\
$\geq 18$ anos & $03(0.7)$ \\
Idade (anos) & $15.5 \pm 1.7$ \\
a, média \pm desvio padrão. & \\
* a idade máxima: 20 anos. &
\end{tabular}

Quanto à etnia, foi possível observar que o uso de SPA apresentou diferença entre os grupos apenas na raça branca, que foi associada a menor frequência de uso $(16,1 \%$ x 21,1\%; $\mathrm{p}=0,03)$. A Tabela 3 detalha o padrão de uso por etnia.

Tabela 3 - Análise do perfil étnico dos pacientes usuários e não usuários de SPA

\begin{tabular}{l|l|l|l}
\hline Etnia & $\begin{array}{c}\text { Uso de SPA } \\
\text { presente } \\
\boldsymbol{n}(\%)\end{array}$ & $\begin{array}{c}\text { Uso de SPA } \\
\text { ausente } \\
\boldsymbol{n}(\%)\end{array}$ & $\boldsymbol{P}$ \\
\hline Branco & $61(16,1)$ & $362(21,1)$ & 0,03 \\
\hline Pardo & $281(74,1)$ & $1228(71,6)$ & 0,33 \\
\hline Negro & $37(9,8)$ & $121(7,1)$ & 0,07 \\
\hline Amarelo & $0(0,0)$ & $03(100)$ & 1,00 \\
\hline
\end{tabular}

$\mathrm{O}$ teste $\mathrm{X}^{2}$ de Pearson assintótico ou o teste exato de Fisher foi utilizado para comparar as variáveis categóricas conforme apropriado.
Quanto aos responsáveis pelos menores, observouse que a maior parte dos indivíduos que utilizam SPA possui apenas a mãe como responsável $(31,4 \%)$, seguida por pais biológicos $(28,4 \%)$ e adolescentes que estavam cumprindo alguma medida socioeducativa (24,8\%). Em contraste, pacientes que não utilizam SPA possuem, em sua maior parte, ambos os pais biológicos como responsáveis $(42,6 \%)$ ou apenas mãe $(32,2 \%)$. Além disso, quando comparados os grupos em uso e sem uso de SPA, houve menor declaração de uso de SPA entre os que tinham como responsáveis os pais biológicos e os que não estavam em medida socioeducativa $(28,4 \times 42,6 \%, \mathrm{p}<0,001$ e $24,8 \%$ x $7,4 \% \mathrm{p}<0,001$, respectivamente).

Com relação à presença de distúrbios psiquiátricos, $87 \%$ dos indivíduos que utilizam SPA possuem algum diagnóstico psiquiátrico, enquanto esse valor cai para $57,1 \%$ ao analisar os que não fazem uso $(\mathrm{p}<0,001)$. A relação entre o uso de SPA e a presença de distúrbios psiquiátricos pode ser verificada na Tabela 4.

Quanto à frequência escolar, foi notado que apenas $47,9 \%$ dos usuários de SPA frequentavam a escola no período de realização dos atendimentos, contra $84,6 \%$ dos não usuários (Figura 2).

Tabela 4 - Relação entre uso de SPA e presença de distúrbios psiquiátricos

\begin{tabular}{lccc}
\hline Presença de distúrbios psiquiátricos & $\begin{array}{c}\text { Uso de SPA presente } \\
\mathbf{n}(\mathbf{\%})^{*}\end{array}$ & $\begin{array}{c}\text { Uso de SPA ausente } \\
\mathbf{n}(\mathbf{\%}) * *\end{array}$ & P \\
\hline Depressão & $92(23.0)$ & $320(17.3)$ & 0.007 \\
Transtorno afetivo bipolar & $10(2.5)$ & $31(1.7)$ & 0.26 \\
Transtornos afetivos não especificados & $23(5.8)$ & $60(3.2)$ & 0.02 \\
Psicose não orgânica não especificada & $30(7.5)$ & $75(4.0)$ & 0.003 \\
Transtornos psicóticos agudos e transitórios & $27(6.8)$ & $72(3.9)$ & 0.01 \\
Esquizofrenia & $12(3.0)$ & $47(2.5)$ & 0.60 \\
Ansiedade & $03(0.8)$ & $54(2.9)$ & 0.008 \\
Reações ao estresse & $29(7.2)$ & $82(4.4)$ & 0.02 \\
Transtornos dissociativos & $04(1.0)$ & $34(1.8)$ & 0.29 \\
Transtorno obsessivo compulsivo & $02(0.5)$ & $77(0.4)$ & 0.67 \\
Transtorno d déficit de atenção e hiperatividade & $13(3.2)$ & $44(2.4)$ & 0.40 \\
Transtorno desafiador de oposição & $09(2.2)$ & $96(5.2)$ & 0.88 \\
Outros transtornos de conduta & $63(15.8)$ & $48(2.6)$ & $<0.001$ \\
Transtornos mistos de conduta e das emoções & $15(3.8)$ & $170(9.2)$ & 0.20 \\
Déficit intelectivo (Retardo Mental) & $27(6.8)$ & $78(4.2)$ & 0.12 \\
Transtornos globais do desenvolvimento & $01(0.2)$ & $03(0.2)$ & $<0.001$ \\
Transtorno de habilidades escolares & $01(0.2)$ & 0.54 \\
\hline
\end{tabular}

* Porcentagem de pacientes que possuem a doença entre todos $(n=400)$ os que utilizam SPA.

** Porcentagem de pacientes que possuem a doença entre todos $(\mathrm{n}=1855)$ os que não utilizam SPA. 


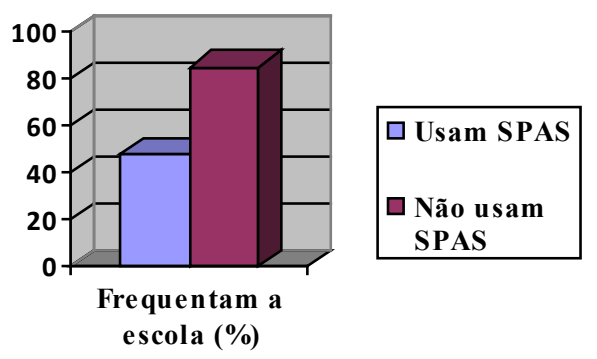

Figura 2 - Porcentagem de jovens usuários e não usuários de drogas que frequentam a escola

\section{DISCUSSÃO}

Observa-se que, na amostra, o perfil de uso de SPA por sexo e faixa etária foi semelhante ao encontrado por Silva et al. ${ }^{9}$, que avaliou esse uso entre estudantes do ensino médio, sendo mais frequente em indivíduos do sexo masculino com idade entre 16 e 17 anos. É importante ressaltar que, na instituição avaliada, os pacientes são encaminhados a outros serviços quando completam dezoito anos, o que justifica a pequena porcentagem de usuários a partir dessa idade. $O$ estudo não avaliou a quantidade e a frequência utilizada, mas somente o uso declarado como queixa no acolhimento/atendimento de urgência. Dessa forma, muitos dos usuários que procuraram o serviço por outro motivo não foram identificados.

No que se refere ao sexo, os meninos apresentaram maior prevalência de uso que as meninas. Nesse quesito, a literatura é variável, com alguns estudos demonstrando inexistência de diferença entre os $\operatorname{sex}^{2,10}$, outros observando maior prevalência no sexo masculino ${ }^{11,12}$ e estudo recente realizado no Brasil revelou maior prevalência em meninas ${ }^{13}$. Uma hipótese levantada é o fato do uso de álcool ser culturalmente mais aceito no sexo masculino, sendo que, nos últimos anos, há relatos de que o comportamento feminino em relação ao uso de substâncias lícitas e ilícitas está se tornando bastante semelhante ao dos homens ${ }^{10}$. Quanto à etnia, foi observada relação inversa de uso com a raça branca, o que pode estar relacionado às diferenças socioeconômicas encontradas no país, uma vez que o uso de substâncias parece estar relacionado com o grau de marginalização da população ${ }^{13}$. Esse resultado contrasta com estudos encontrados, sendo que outros autores brasileiros não observaram diferença entre as diferentes etnias ${ }^{2,14,15}$.

Foi identificada prevalência de $17,7 \%$ de atendimentos onde houve relato de uso de SPA, sendo observado uso de maconha (14\%), cocaína (7\%), álcool $(5,7 \%)$, tabaco $(4,2 \%)$, solventes e inalantes $(3,6 \%)$ e crack $(1,7 \%)$. O uso de álcool e tabaco contrasta com a maior parte dos estudos encontrados, tanto brasileiros quanto estrangeiros, que observam consumo consideravelmente maior. Bertoni et al. ${ }^{16}$, em estudo realizado em Minas Gerais, observou prevalência de uso de álcool em jovens de 10 a 19 anos de $45,9 \%$ e tabaco $23 \%$. Silva et al. ${ }^{9}$, em São José do Rio Preto (SP), observaram frequência de uso de álcool de $77 \%$ e tabaco $28 \%$. Elicker et al. ${ }^{15}$, em Porto Velho (RO), observou prevalência de $24 \%$ para álcool e $6,4 \%$ de tabaco em população de 05 a 18 anos. Lopes et al. ${ }^{11}$ afirmam que o álcool é a droga mais utilizada no Brasil, dado que corrobora o encontrado em outros países ${ }^{17,18}$, contrastando com os achados do presente estudo, em que a droga ocupa terceiro lugar em frequência de uso. Essa diferença pode estar relacionada ao fato dos dados terem sido obtidos através de análise de prontuário de atendimentos de urgência, onde a anamnese costuma ser mais objetiva, em contraste com os demais estudos que foram realizados em escolas e os alunos foram questionados especificamente sobre o uso de drogas. Assim, não é possível saber, por exemplo, se durante o atendimento do plantão não foi investigado o uso dessas substâncias. Ademais, a faixa etária do presente estudo é mais ampla, abrangendo também crianças pequenas, onde, geralmente, não se faz tal investigação.

Quanto ao uso de maconha, o estudo encontrou prevalência semelhante à encontrada por Silva et $\mathrm{al} .{ }^{9} \mathrm{em}$ São José do Rio Preto (SP) (18,1\%), e Vieira et al. ${ }^{2}$ no Sul do Brasil (16,9\%), porém discretamente mais baixa que encontrada por Nascimento et al. ${ }^{19}$ em Guarulhos (SP) (28\%). Novamente, o que pode ser explicado pela amostra do estudo atual ser de um serviço de urgência. Estudos realizados em Estados Unidos e América Central revelaram prevalência mais baixa, respectivamente $7,4 \%$ e $4 \%{ }^{12,20}$.

A literatura relata que a proporção de jovens que experimentaram drogas ilícitas ao menos uma vez, em diferentes países, foi: superior a $40 \%$ na Austrália, maior que $35 \%$ no Canadá e maior que $40 \%$ nos Estados Unidos $^{1}$. Esses dados contrastam com a menor prevalência de uso encontrada no estudo $(17,7 \%)$, que pode ter sido subestimada, uma vez que o jovem não foi questionado especificamente quanto à experiência com a droga, devido à metodologia empregada pelo estudo.

$\mathrm{O}$ estudo observou que a doença psiquiátrica mais prevalente foi depressão, com prevalência de $23 \%$ em usuários de SPA e $17 \%$ em não usuários. Houve prevalência superior no grupo em uso de SPA de outras condições como: psicose não orgânica não especificada, transtornos afetivos não especificados, reações ao estresse, transtornos psicóticos agudos e transitórios e outros transtornos de conduta. Esses resultados reforçam os dados estabelecidos na literatura, que demonstram relação de ocorrência simultânea desses distúrbios ${ }^{1,13}$. No presente estudo não foi observada relação entre uso de SPA e transtorno afetivo bipolar, o que contrasta com a associação entre ambas já bem estabelecida na literatura ${ }^{21}$. $\mathrm{O}$ uso de drogas pode estar envolvido como um fator de risco, como 
consequência dessas condições, ou até mesmo determinar maior gravidade em casos de doença psiquiátrica prévia ${ }^{1}$. Recente estudo publicado por Gobbi et al. ${ }^{22}$ em 2019 identificou que o consumo de maconha por adolescentes foi associado a um risco aumentado de desenvolver depressão e comportamento suicida mais tarde na vida, mesmo na ausência de uma condição pré-mórbida. Esse dado aumenta a preocupação com a elevada prevalência do uso dessa substância encontrada no estudo e realça a importância de criação de medidas para combatê-lo, principalmente levando em consideração que a prevalência real de uso provavelmente é ainda maior.

Quanto à estrutura familiar, 28,4\% dos usuários de SPA tinham como responsáveis ambos os pais biológicos, comparado a uma porcentagem de $42,6 \%$ dos não usuários. Apesar de o presente estudo ser um corte transversal e não permitir o estabelecimento de relação causal, tal resultado sugere que a falta de suporte parental pode predispor a um maior consumo de drogas entre adolescentes, dado corroborado por Zappe et al. ${ }^{23}$. Broeker et al. ${ }^{24}$ observaram que práticas educativas parentais socialmente desejáveis se associam a menor dependência química. Esses dados podem servir de substrato para encorajar os profissionais que lidam com esses pacientes a estender o atendimento aos seus responsáveis, conscientizando-os através de práticas psicoeducativas sobre a importância dos vínculos familiares fortalecidos na prevenção ao uso de substâncias psicoativas por parte de seus filhos. Não foram encontrados, porém, artigos que analisassem de forma direta a presença de ambos os pais biológicos como fator de proteção.

Apesar de a literatura apontar que a criação apenas de pai ou mãe é um fator de risco para uso de substâncias psicoativas ${ }^{23}$, não foi encontrada, na análise em questão, diferença estatística em relação a responsável único quando comparados grupos em uso de SPA e não usuários. Tal diferença pode não ter sido encontrada visto que alguns dos atendimentos tinham como responsável, no momento do atendimento, unidades socioeducativas sem informações sobre quem era o responsável pelo menor antes do acautelamento. Assim, esse fator pode ter sido um viés ao avaliarmos a variável "responsável pelo menor", não nos permitindo fazer conclusões a respeito da estrutura familiar com clareza.

Além disso, houve maior prevalência de unidade socioeducativa como responsável entre os usuários de SPA (24,8\%) que nos não usuários (7,4\%). Essa associação já foi relatada, com prevalência de até $75 \%$ de uso de SPA em adolescentes em conflito com a lei de acordo com dados de 2012 do Conselho Nacional de Justiça. Além disso, a maioria deles utiliza mais de um tipo de substância psicoativa e grande parte dos jovens em instituições socioeducativas está ligada ao tráfico de drogas ${ }^{25}$.

Estudos apontam que o próprio consumo de drogas é um fator predisponente para cometer uma conduta infracional, visto que algumas dessas substâncias (como cocaína, álcool, inalantes) podem levar a aumento de agressividade $^{26}$. Foi observado na literatura que o uso precoce de substâncias psicoativas está relacionado a uma maior probabilidade de cometer atos delinquentes precocemente ${ }^{25}$.

Devido à utilização de dados de prontuários médicos como fonte das informações do estudo, algumas limitações podem ser destacadas. Alguns arquivos de prontuários de pacientes atendidos durante o período do estudo foram extraviados ou não encontrados, inviabilizando sua coleta. Outro aspecto limitante observado foi a presença de dados ilegíveis e/ou incongruentes com o contexto, que tiveram que ser excluídos da pesquisa. Além disso, as informações contidas são decorrentes de uma entrevista em atendimento de acolhimento e/ou urgência e, nem sempre, todas as informações do roteiro estavam contidas na avaliação, pois muitas vezes os pacientes não são questionados especificamente pelo uso de SPA. Os atendimentos de urgência demandam uma condução rápida podendo ocasionar registro resumido de alguns dados solicitados pelo roteiro. Dessa forma, a prevalência do uso de SPA pode ter sido subestimada.

\section{CONCLUSÃO}

O presente estudo observou significativa prevalência no uso de drogas entre crianças e adolescentes, reforçando os dados disponíveis na literatura atual $1^{1,2,3,4}$. É possível presumir que a real prevalência seja maior, uma vez que se acredita que a porcentagem real de jovens que utilizam essas substâncias é maior que a parcela que chega ao serviço de urgência. Além disso, é provável que nem todos os atendimentos tenham investigado o uso de SPA. Tendo em vista os resultados, é importante que os serviços de urgência que atendem a tal população investiguem o uso de SPA principalmente quando não é a queixa principal visando identificação precoce para psicoeducação.

Foram identificadas associações com doenças psiquiátricas, em especial a depressão, e com a ausência de pais como responsáveis, corroborando a maior parte dos estudos encontrados. Ressaltamos a necessidade de maiores estudos com a mesma população para tentar estabelecer se o uso da droga seria prévio ou posterior ao transtorno psiquiátrico.

Sabe-se que o uso de drogas pode comprometer o futuro dos jovens, trazendo prejuízos sociais e econômicos, além de aumentar os níveis de violência e as taxas de homicídios no país. Dessa forma, os resultados encontrados demonstram a necessidade de intervir nas populações de maior risco e no ambiente em que estão inseridas. A elevada prevalência do uso em adolescentes que estão cumprindo medida socioeducativa, por exemplo, pode sugerir a necessidade de abordagens nesses locais.

Não é de nosso conhecimento outro estudo que aborde um período de um ano em serviço de urgência 
infantil. Além disso, a importância de um estudo realizado na urgência, onde somente foi considerado uso de SPA se isso fosse trazido como queixa pelo menor ou responsável, é a comparação com estudos em que foi investigado o uso geral pelos jovens. Dessa forma, será possível avaliar a diferença entre o real uso de SPA e quando isso passa a ser considerado um problema para o jovem ou responsável. Por ser um estudo pioneiro em tal local de atendimento, ressalta-se a necessidade de novos estudos em outras regiões do país antes de uma generalização dos dados.

Declaração de responsabilidade e conflito de interesses: Declaramos autoridade pelo conteúdo do manuscrito e inexistência de conlitos de interesse.

Contribuição dos autores: ACMC: Elaboração do projeto de pesquisa, coleta de dados, pesquisa do referencial teórico em bases de dados, redação e adequação do artigo às normas da revista com posterior submissão à plataforma para publicação. ACBMF: Elaboração do projeto inicial, pesquisa do referencial teórico em bases de dados, coleta de dados, redação do artigo. ALSG; AMFC: elaboração do projeto inicial, coleta de dados. AMSL; JPVT; CPC; MSN: Coleta de dados. LRC; MCLM: Orientações acerca da elaboração do projeto inicial, desenvolvimento do projeto e do roteiro de coleta de dados; financiamento dos gastos.

Agradecimento: agradecemos a DAV pelo auxílio estatístico e ao Centro Psiquico da Adolescência e Infância (CEPAI), instituição pertencente À Fundação Hospitalar do Estado de Minas Gerais (FHEMIG).

\section{REFERÊNCIAS}

1. Bukstein O. Substance use disorder in adolescents: Epidemiology, pathogenesis, clinical manifestations, course, assessment, and diagnosis. UptoDate, 2019. Available from: https://www.uptodate.com/contents/ substance-use-disorder-in-adolescents-epidemiologypathogenesis-clinical-manifestations-and-consequencescourse-assessment-and-diagnosis.

2. Vieira PC, Aerts DRGC, Freddo SL, Bittencourt A, Monteiro L. Uso de álcool, tabaco e outras drogas por adolescentes escolares em município do Sul do Brasil. Cad Saúde Pública. 2008;24(11):2487-98. https://www.scielosp. org/article/csp/2008.v24n11/2487-2498/.

3. Carlini ELA, Noto AR, Sanchez VDM, Carlini, CMA, Locatelli, DP, Abeid LR, et al. VI Levantamento Nacional sobre o Consumo de Drogas Psicotrópicas entre Estudantes do Ensino Fundamental e Médio das Redes Pública e Privada de Ensino nas 27 Capitais Brasileiras. São Paulo: CEBRID; UNIFESP; 2010. Disponível em: https://www. cebrid.com.br/vi-levantamento-estudantes-2010/.

4. McArdle P. Use and misuse of drugs and alcohol in adolescence. BMJ. 2008;337(7660):46-50. doi: https:// dx.doi.org/10.1136/bmj.a306.

5. United Nations Organizaton (UNO). Drugs crime and viollence: the microlevel impact. New York: UNO; 2004. p.11. Available from: https://www.incb.org/documents/ Publications/AnnualReports/Thematic_chapters/English/ AR_2003_E_Chapter_I.pdf.

6. Drummond EF, Souza HNF, Hang-Costa TA. Homicídios, álcool e drogas em Belo Horizonte, Minas Gerais, Brasil, 2000-2009. Epidemiol. Serv Saúde (Brasília). 2015,24(4):607-16. doi: https://dx.doi.org/10.5123/S167949742015000400003.

7. World Health Organization. Management of substance abuse. terminology \& classification [cited 2020 March 22]. Available from: https://www.who.int/substance_abuse/ terminology/psychoactive_substances/en/.

8. Garcia F. Manual de abordagem de dependências químicas. Belo Horizonte: Utopika Editorial; 2014.
9. Silva EF, Pavani RAB, Moraes MS, Neto FC. Prevalência do uso de drogas entre escolares do ensino médio do Município de São José do Rio Preto, São Paulo, Brasil. Cad Saúde Pública (São Paulo). 2006;22(6):1151-8. doi: https://dx.doi. org/0.1590/S0102-311X2006000600004.

10. Wang J, Simons-Morton BG, Farhart T, Luk JW. Sociodemographic variability in adolescent substance use: mediation by parents and peers. Prev Sci. 2009; 10(4):38796. doi: https://dx.doi.org/10.1007/s11121-009-0141-1.

11. Lopes GM, Nobrega BA, Del Prette G, Scivoletto S. Use of psychoactive substances by adolescents: current panorama. Rev Bras Psiquiatr (São Paulo). 2013;35(1):S51-S61.doi: https://dx.doi.org/10.1590/1516-4446-2013-S105.

12. Dormitzer, CM, Gonzalez GB, Penna M, Bejarano J, Obando P, Sanchez M. The PACARDO research project: youthful drug involvement in Central America and the Dominican Republic. Rev Panam Salud Pública. 2004;15:400-16. doi: https://dx.doi.org/10.1590/s1020-49892004000600006.

13. Benner AD, WangY. Adolescent substance use: the role of demographic marginalization and socioemotional distress. Dev Psychol. 2015;51(8):1086-97. doi: https://dx.doi. org/10.10370000026.

14. HortaI RL, MolaII CL, HortaII BL, MattosI CNB, Andreazzi MA, Oliveira-Campos M, et al. Prevalência e condições associadas ao uso de drogas ilícitas na vida: Pesquisa Nacional de Saúde do Escolar 2015. Rev Bras Epidemiol (Porto Alegre). 2018;21(sup.1):e180007. doi: https://dx.doi.org/10.1590/1980-549720180007.

15. Elicker E, Palazzo LS, Aerts DRGC, Alves GG, Câmara S. Uso de álcool, tabaco e outras drogas por adolescentes escolares de Porto Velho-RO, Brasil. Epidemiol Serv Saúde (Brasília). 2015;24(3):399-410. doi: https://dx.doi. org/10.5123/S1679-49742015000300006.

16. Bertoni N, Bastos FI, Mello MB, Makuch MY, Sousa MH, Osis MJ, et al. Uso de álcool e drogas e sua influência sobre as práticas sexuais de adolescentes de Minas Gerais, Brasil. Cad Saúde Pública (Rio de Janeiro). 2009;25(6):1350-1360. doi: https://dx.doi.org/10.1590/ S0102-311X2009000600017.

17. HortaI RL, MolaII CL, HortaII BL, MattosI CNB, 
Andreazzi MA, Oliveira-Campos M, et al. Prevalência e condições associadas ao uso de drogas ilícitas na vida: Pesquisa Nacional de Saúde do Escolar 2015. Rev Bras Epidemiol. (Porto Alegre). 2018; 21(1). doi: https://dx.doi. org/10.1590/1980-549720180007.

18. European Monitoring Centre for Drugs and Drug Addiction (EMCDDA). European School Survey Project on Alcohol and Other Drugs (ESPAD). ESPAD Report 2015: results from the European School Survey Project on Alcohol and Other Drugs. Lisboa, Portugal; 2015. Available from: http:// www.espad.org/sites/espad.org/files/ESPAD_report_2015. pdf.

19. Nascimento MO, Avallone, DM. Prevalência do uso de drogas entre adolescentes nos diferentes turnos escolares. Adolesc Saude. 2013;10(4):41-49. Disponível em: https:// cdn.publisher.gn1.link/adolescenciaesaude.com/pdf/ v10n4a06.pdf.

20. Center for Behavioral Health Statistics and Quality. Behavioral health trends in the United States: Results from the 2014 National Survey on Drug Use and Health (HHS Publication No. SMA 15-4927, NSDUH Series H-50). Rockville, MD; 2015. Available from: https://www.samhsa. gov/data/sites/default/files/NSDUH-FRR1-2014/NSDUHFRR1-2014.pdf.

21. Duffy A, Horrocks J, Milin R, Doucette S, Persson G, Grof P. Adolescent substance use disorder during the early stages of bipolar disorder: a prospective high-risk study. J Affect Disord. 2012;142(1-3):57-64. doi: https://dx.doi. org/10.1016/j.jad.2012.04.010.
22. Gobbi G, Atkin T, Zytynski T, Wang S, Askari S, Boruff $\mathrm{J}$, et al. Association of Cannabis Use in Adolescence and Risk of Depression, Anxiety, and Suicidality in Young Adulthood: a systematic review and meta-analysis. JAMA Psychiatry. 2019;76:426. doi: https://dx.doi.org/10.1001/ jamapsychiatry.2018.4500.

23. Zappe, JG; Dapper, F. Drogadição na Adolescência: Família como Fator de Risco ou Proteção. Rev Psicol IME (Passo Fundo). 2017;9(1):140-58. doi: https://dx.doi. org/10.18256/2175-5027.2017.v9i1.1616.

24. Broecker CZ, Jou GI. Práticas educativas parentais: a percepção de adolescentes com e sem dependência química. Psico-USF (Porto Alegre). 2007;12(2):269-79. Disponível em: http://pepsic.bvsalud.org/pdf/psicousf/ v12n2/v12n2a15.pdf.

25. Gallo AE, Williams LCA. Adolescentes em conflito com a lei: uma revisão dos fatores de risco para a conduta infracional. Psicol Teor Prat (São Paulo). 2005;7(1):181-91. https://www.scielo.br/pdf/ptp/v28n2/06.pdf.

26. Scomparini LB, Santos B, Rosenheck RA, Scivoletto S. Association of child maltreatment and psychiatric diagnosis in Brazilian children and adolescents. Clinics (São Paulo). 2013;68(8):1096-102. doi: https://dx.doi.org/10.6061/ clinics/2013(08)06.

Submetido: 08.01.2020

Aceito: 11.08.2020 